\title{
Whitman y el Anti-modernismo
}

$\mathrm{F}_{\mathrm{N}}$ 1937, ante este mismo grupo ${ }^{1}$ que no se habia hecho independiente todavía de la sección-madre dedicada a la literatura contemporánea española e hispanoamericana, tuve el privilegio de abordar por primera vez el tema de Whitman en la' América hispana. Expliqué entonces ${ }^{2}$ que aunque leido, traducido, apreciado $y$ hasta imitado por los grandes valores modernistas, Darío, Lugones, Chocano y Nervo, Whitman no podía figurar en la compañía de "los raros" exaltados por los modernistas de la época de Prosas profanas como los dioses del arte por el arte. Como bien lo dijo Fernando Alegria en un artículo posterior al mío, "es la voz de Whitman lo que está presente a través de todo el movimiento modernista; no su espiritu". 3

$\mathrm{Y}$ señalé que el verdadero significado del arte y del mensaje del Gran Viejo no llegó a ser plenamente comprendido en la América española sino hasta que se iniciara la reacción contra el pesimismo finisecular, contra el verbalismo musical y decorativo, y contra la actitud aristocrática y anti-social de esa fase del modernismo que se iba a denunciar como rubendariana. Terminé afirmando que desde principios del siglo Walt Whitman ha venido a ser una de las promesas espirituales y estéticas que más hondamente han conmovido el arte y el corazón de la juventud hispanoamericana.

No es mi propósito hoy delinear el impacto del espíritu de Whitman sobre la literatura hispanoamericana de 1905 en adelante; ni defender con respecto a la América hispana la afirmación de Luis Franco: que "de ningún otro hombre vivo o muerto puede hablarse con tanta actualidad como de Whitman". " Esta "contemporaneidad efectiva" de Whitman es muy conocida para precisar de aclaraciones o de amplificación aquí; alli están los trabajos de apreciación 
crítica de Luis Franco, ${ }^{5}$ José Gabriel, ${ }^{6}$ Juan Felipe Toruño, 7 y otros; y las traducciones al español de algunos de los mejores estudios sobre Whitman publicados aquí en recientes años, como, por ejemplo, los de Caneron Rogers, ${ }^{8}$ de Frances Winwar, ${ }^{9}$ y de Babette Deutsch ; ${ }^{10}$ y las traducciones de Rodolfo Usigli ${ }^{11}$ y de León Felipe ${ }^{12}$ entre otras ${ }^{13}$ para probar hasta qué punto Whitman sigute colaborando con la América de hoy "en busca de su expresión". ${ }^{14}$

Menos conocido es el papel desempeñado por Whitman en la reacción contra el modernismo. $\mathrm{Y}$ son pocos los que como Suárez Calimano se han referido a la influencia de Whitman sobre los poetas que iniciaron tal reacción, ${ }^{15}$ los que requerían "un nuevo impulso original, una nueva oleada de vida que renovara el sentido de la realidad y que creara nuevos valores estéticos". Alberto Zum Felde, analizando las distancias que apartan al europeo Verhaeren "futurista" y "crepuscular" "del viejo Adam mojado del rocio de la aurora", afirma que fué "del seno rudo de Manhattan" que vino "al fatigado mundo occidental, el impulso renovador". ${ }^{16} \mathrm{Y}$ fué Alvaro Armando Vasseur, compatriota de Zum Felde, quien terminó èl prólogo a sus traducciones de Whitman publicadas en 1912, con esta profética invocación: “iBendita sea la tempestad de su arte, si logra airear la atmósfera literaria hispanoamericana, tan recargada de emanaciones gallináceas!" 17

Espíritu inquieto y rebelde, a Vasseur le atraen una diversidad de autores extranjeros, entre ellos Poe y Whitman. A éste confiesa que le debe "diversas y profundas sugestiones". ${ }^{18}$ Es difícil determinar hasta qué punto Whitman le habrá inspirado en sus ideales de transformación social y su esfuerzo de lograr una nueva expresión poética - la cual nunca realizó. En todo caso parece innegable que existe una relación significativa entre su descubrimiento y aprecio de Whitman y su reacción contra la estética modernista manifestada en los Cantos augurales (1904) y los Cantos del Nuevo Mundo (1906). Vasseur ocupa, pues, un lugar primordial en el caso de Whitman en América, habiendo sido el primero en ofrecer al público hispano una colección de versos de Whitman en español y uno de los primeros poetas hispanoamericanos en rebelarse contra el modernismo.

Los primeros frutos de esta labor misionera de Vasseur no tardan en manifestarse en pleno Montevideo. En el mismo año en 
que Vasseur publica sus traducciones de Whitman (1912), ${ }^{19}$ salen los primeros versos de la pluma "exuberante y vital" del uruguayo Carlos Sabat Ercasty, joven "fuerte, alto, de mirada azulada... con una sonrisa siempre dibujada en los labios", ${ }^{20}$ que hasta en el aspecto físico se parece mucho a Whitman. Recogidos en su primer libro Pantheos publicado en 1917, estos versos no revelan nada nuevo ni original. Pero en los años que corren entre 1912 y 1917; Sabat Ercasty debe haber descubierto a Whitman - y con toda probabilidad el Whitman de Vasseur. Que yo haya podido averiguar, el poeta nunca nos declara en ninguna parte haber sido discípulo de Whitman. Ni siquiera hay en toda su obra referencia alguna a Walt, como tampoco las hay a otras fuentes literarias. De primera vista su poesía está libre de toda influencia libresca; pero aun así, es imposible negar su parentesco con Whitman ni dudar de que le debe mụcho en la técnica y en la temática de su arte. Desde "Ura- . nia", escrito en 1916, que es el primer poema en que emplea una técnica whitmaniana, Sabat Ercasty seguirá manifestando por más de una década su preferencia por la prosa poética y el verso libre $\mathrm{y}$ por los catálogos y las repeticiones de palabras y frases iniciales que asociamos con Whitman. $Y$ cantará a toda voz sus inclinaciones cósmicas, la sagrada misión del poeta, la inseparabilidad del cuerpo y del alma, y su rebelión contra "la arrogancia desdeñasa" de la literatura. Oigámosle cuando en "El hombre" parece que es el poeta de Hojas de hierba que nos habla:

"Yo reposaba tendida sobre la madre tierra, en este dulce día de otoño en que me sentí lleno de Dios y comencé a escribir el poema que ofrezco a mis hermanos", ${ }^{21}$ y cuando asi se dirige "Al poeta que viene", exhortándole a que se rebele contra las musas modernistas:

¡Poeta! ; Cumple desde hoy con tu sagrado ministerio! Abandona tus musas gemidoras y tus concubinas neuróticas y flageladas por todas las lepras de la carne y del espíritu.

Aleja tus plantas aladas de los jardines enanos donde el artificio de las retóricas trunca todos los días la inmensa aspiración de la naturaleza... Y deja, deja para siempre la torre de marfil que te aparta de los hombres, para quienes has nacido y para los que no gozas el derecho de negarte, pues se incuba en tu frente el destino del más alto sacerdocio. 22

$\mathrm{Y}$ así como Whitman en su llamada a los "Poetas por venir" ("Poets to Come") les exalta como "raza nueva, continental, atlética, nativa, 
más grande que las antes conocidas", también Sabat Ercasty a la "juventud temeraria" que ha de llevar "como una gran bandera llameante el incendio de la inquietud para todos los espíritus":

Hijo de un mundo que nace, tienes como destino lá tarea creadora de un Dios. No has aparecido en América para repetir la obra de las razas caducas... Tabla rasa con todo, joven de los veinte años! Un furor divino debe agitar tus miembros con el más hondo esfuerzo, en tanto que aniquilas todas las ruinas que te of rece el orgullo de los viejos continentes. Entrégate a la acción gozoso y magnífico, con la visión lejana del triunfo. ${ }^{23}$

De los postmodernistas es Sabat Ercasty el poeta que quizá más se parece a nuestro Whitman. $E$ indudablemente también a él, asi como a Vasseur se le debe no poca de la admiración por Whitman que han sentido otros uruguayos, Fernán Silva Valdés ${ }^{24}$ y Juan Parra del Riego ${ }^{25}$ entre ellos, por citar sólo dos.

Bien sabido es que en unas regiones de América el modernismo no llegó a florecer sino tardíamente, mientras que en otras continuó cultivándose mucho más allá de 1905, fecha establecida como principio de la reacción postmodernista. Puerto Rico y Venezuela representan, respectivamente, estas dos divergencias con relación al desarrollo normal del movimiento. En estos dos países, por lo tanto, inicióse algo más tarde la reacción - y en ésta también juega Whitman un papel considerable.

En 1944 murió en Puerto Rico el poeta más fuerte y más original que haya cantado allí en lengua española. "Poeta isleño con visión continental", ${ }^{26}$ Luis Lloréns Torres nació a la vida literaria en el mismo momento en que el modernismo empezaba a imperar en las letras americanas. Pero estos años los pasó en España donde publicó los dos libros de que consta la primera fase de su desenvolvimiento artístico, uno en prosa titulado América (1898), el otro en verso titulado Al pie de la Alhambra (1899), que poco tienen que ver con su obra madura posterior. De vuelta de España, inmediatamente después del '98, se dedica a la nueva vida politica isleña, no volviendo a un cultivo serio de la poesía hasta el año 1913 cuando empieza a redactar, con otros, la importante Revista de las Antillas (marzo, 1913-julio, 1914), época del apogeo del modernismo en la isla. Si en el principio se revela como modernista tardío de la última etapa de Darío, ya antes de la guerra europea se aparta del movimiento con aspiraciones renovadoras, queriendo desplazarlo, "en- 
sayando una nueva técnica lejos de toda tiranía oficiosa". ${ }^{27} \mathrm{Al}$ definir su nueva modalidad poética, que llama "pancalismo", que quiere decir "todo es bello", insinúa en el poema "Visión de la naturaleza" que su estética renovadora coincide en mucho con la de Whitman:

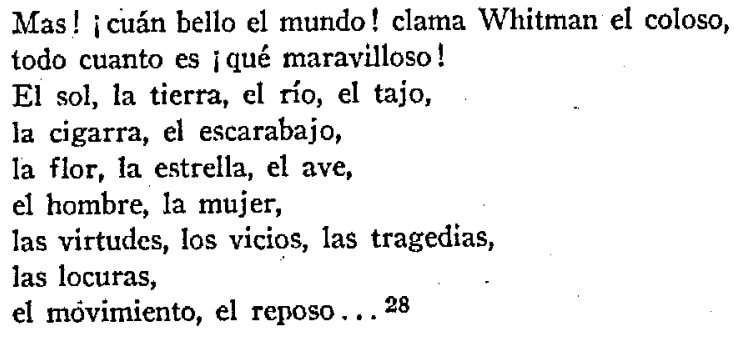

$Y$ en su "Visión del ritmo" se declara hasta "más libre que Whitman en la selva americana".

Antonio S. Pedreira y Concha Meléndez encuentran marcada influencia de Whitman en los poemas "Velas épicas" y "La canción de las Antillas", que son de la segunda y mejor época de Lloréns, es decir, de los años 1913-1915, el momento en que logra su expresión más definitiva. En el primero se la ve principalmente "en la construcción estrófica, en el encadenamiento de las ideas, a veces series de enumeraciones", $y$ en la fuerza whitmaniana que respira todo el poema; en el segundo se la ve, además, en el "cosmopolitismo fraterno" y en el "afán de salud espiritual y física que hizo cantar al poeta de Manhattan:

Give me fresh corn and wheat, give me serene-moving animals teaching content Give me solitude, give me Nature, give me again, O Nature your primal (sanities!" 29

Dícese que Lloréns negó haber imitado a Whitman - aunque habiendo confesado al mistno timpo que le gustó mucho. ${ }^{30}$ Sea eso como fuera, es innegable que lo que representa el whitmanismo en la isla, lo expresa Lloréns que ha dicho este gran decir whitmanista: "No hay prosa, no hay más que verso, toda prosa es verso." Además, no cabe duda de que consciente o inconscientemente Lloréns se endeudó no poco para con Whitman en la ideación de su estética pancalista. 
El primer paso definitivo de la reacción isleña contra el modernismo, el pancalismo, se destaca por no haber dejado ningún discípulo ni ninguna resonancia, por desconocido, y por haber sido precursor de los mil "ismos" de postguerra. En cambio, el whitmanismo introducido en Puerto Rico como aspecto del pancalismo y como una de las facetas dominantes de la estética de su fundador y único cultivador, prosperó, no faltando en los años posteriores otros poetas de la tierra de Lloréns que se han declarado sinceros admiradores, si no discípulos, del bardo de Camden, entre ellos Pedro Juan Labarthe, ${ }^{31}$ Luis Muñoz Marín, ${ }^{32}$ y Fran Cervoni Brenes. ${ }^{33}$

En Venezuela el modernismo continúa dominando el ambiente estético y espiritual hasta años después de iniciada la reacción en otras partes de la América hispana. No será sino hasta después de la primera Guerra Mundial cuando aparece en Venezuela la nueva generación poética, llamada la "Generación de 1918", a veces el "vanguardismo". Fué ésta la generación que intentó librarse de la larga dominación modernista y madrigalesca. El que dió los primeros pasos seguros hacia una nueva estética venezolana fué el poeta "Antonio Arráiz, cuyo libro Aspero, publicado en 1924, "vino a ser el primer ensayo afortunado de unificación" de la poesía y la realidad venezolanas.

¿Cómo era el jefe de esta nueva generación poética? Arturo Uslar Pietri nos lo describe asi:

"Antonio Arráiz fué una típica irrupción de barbarie sana y arrolladora. Era un mozo atlético, rojo y feo. Carecía de los hábitos inherentes al poeta. No fumaba, no bebía, había leído muy poca literatura. Jugaba football, escribía crónicas deportivas y la publicidad de una empresa de espectáculos, y en los días libres se iba de excursión a los pueblos, a los campos y a los montes." ${ }^{34}$ Se comprenderá, pues, que fué la vida y no la literatura que le sirvió a Arráiz de principal fuente poética. Había, sin embargo, tres maestros literarios _- "tres magníficos maestros de americanidad": Homero, Bufalo Bill y Walt Whitman. $Y$ estos maestros le enseñaron a "expresar las cosas que le parecían esenciales en la forma en que se le presentaban, sin elaboración y sin artificio". El libro Aspero asi nacido no podía ser modelo de métrica; “... tenía que ser áspero, informe, violento y libre como el mundo del que era imagen, e imagen cabal". "Pocos libros como Aspero" —afima Uslar Pietri- 
"han tenido una importancia mayor en la orientación de la conciencia colectiva. Ya para muchos de los venezolanos de hoy [esto fué en 1939, 15 años más tarde] ha dejado de ser un libro para convertirse en un clima de formación del sentimiento y del espíritu". ${ }^{35}$

En la creación y orientación de esta conciencia colectiva venezolana pueden señalarse huellas inspiradoras $\mathrm{e}$ inequívocas de Whitman, tanto directas como indirectas a través del libro revolucionario de Arráiz. Dice Uslar Pietri que sin saberlo éste "se había hecho... creador de una conciencia a la manera de Whitman". Los motivos del poemario son de la más noble simplicidad y de innegable parentesco whitmaniano: la conciencia solitaria ante la naturaleza, la fraternidad para con los vivos y los muertos, el egoísmo, el trabajo, el continente americano en su totalidad. Una vez más es el autor de Las lanzas coloradas quien insiste en las aproximaciones whitmanianas en la obra de Arráiz cuando dice que en Aspero "está cantando a América en la misma actitud en que el otro, el barbudo solitario de los Alleghanys, la está oyendo cantar, con un son áspero y pueril de niño que aprende a nombrar las cosas nuevas". ${ }^{36}$

De los poetas de la Generación de 1918, contemporáneos de Arráiz, y de los que posteriormente se agregaron a la Generación en 1928, como también de los que en 1936 constituyeron el grupo de Vier$n e s$, los hay que declaran o revelan su admiración por Whitman. iUslar Pietri mismo, que tan fuertemente sintió la modalidad whitmaniana en la obra de Arráiz, confiesa deberle algo también a Walt en la época que corre entre 1928 y 1934, época en que su interés en la vida criolla responde vivamente a la atracción americanista de Whitman! Otros admiradores de Whitman han sido Jacinto Fombona-Pachano (véase su poema "Un alerta para Abraham Lincoln"), ${ }^{37}$ Pedro Sotillo, Pedro Garcia Lopenza y Pablo Rojas Guardia. Cabe observar que en Venezuela el reconocimiento de Whitman no queda mayormente reducido al momento de la inauguración de la nueva modalidad estética allá por 1918. En 1945, al declararse libre de la musa de ayer para poder darse frente con la realidad americana de hoy, cantada en su libro En este Nuevo Mundo (Caracas, "Suma", 1945), Otto d'Sola escogerá por lema de sus versos plenamente imbuídos del espiritu de Whitman, estas célebres palabras de Walt : 
palabras que bien pudieran haber servido de lema también para el Aspero de Antonio Arráiz de veinte años atrás.

No cabe ya sino mencionar de paso algunos cuantos nombres identificados en otros paises con la reacción contra el modernismo, nombres que han dejado pruebas también del efecto saludable de los versos emancipadores de Whitman.

Conocido es el ensayo del chileno Armando Donoso sobre Whitman, publicado por primera vez en $1915 .{ }^{38}$ Dirá Torres-Rioseco en el prólogo de su tomo de traducciones de Walt en $1922,{ }^{39}$ que Donoso habia escrito "más de un ensayo sobre Whitman despertando profundo interés entre la gente joven", añadiendo que "en los versos de Pedro Prado y muy en especial en la vigorosa labor del poeta chileno Luciano Morgad, resuena el eco del gigante de Camden". Demasiado cerca de la reacción ya en pleno desarrollo, Torres-Rioseco no pudo ver en aquel momento que era ya la realidad lo que profetizó con respecto a la poesía de Whitman en América, que sería como "una corriente purificadora" para arrastrar "tanta frase hecha, tanta palabra rutinaria" que el modernismo había dejado en la poesía hispanoamericana. Años más tarde, Torres-Rioseco afirmaría su propia deuda a Whitman, declarando que era el poeta que más profundamente le inspiraba en sus años ya de madurez. ${ }^{40}$

No creo yo necesario señalar el fuerte parentesco entre el chileno Pablo de Rokha y Whitman ni recordarles la devoción que sentía el joven Neruda de Poemas de amor, allá por 1920, por el hijo de Manhattan que habia cantado "the body electric". Conviene que Domingo Melfi hable de una vez por los jóvenes chilenos de aquel entonces en confirmación de cuanto ellos debían a los cantos musculosos de la naturaleza que Whitman había arrojado sobre América:

"Esta literatura como de confitería había hecho estragos en las sociedades americanas y un secreto cansancio, un hastío invisible, se insinuaba concreto en la generación joven. Se echaba de menos algo más poderoso, algo más fuerte y real; pasiones más hondas, formas, en fin, viriles de lucha ... Whitman parecia un soplo de las montañas, un grito salvaje de los desiertos ..." 41

En el Perú el grupo rebelde será el que se forma en torno a Colónida. ${ }^{42}$ Serán los que contribuyen a la antología Voces muiltiples publicada en Lima en 1916. ${ }^{43}$ Será el malogrado hijo de Manuel González-Prada, don Alfredo, que leyó Hojas de hierba por primera 
vez a los 20 años de edad, allá en Lima, por 1911-1912, traduciendo "aquel poema de tan jugosa animalidad sexual titulado "A woman waits for me'," cuyo último verso según él, "tiene engastado un 'lovingly' que es un acierto genial". ${ }^{44}$ ¿ Recordarán el verso?

I shall look for loving crops from the birth, life, death, immortality, I plant so lovingly now.

$\mathrm{Y}$ algo más tarde serán Abraham Valdelomar y Alberto Hidalgo. No hay que buscarle a Hidalgo huellas whitmanianas en su época de "futilezas surrealistas", sino más bien en su época anterior de sólida obra poética.

En-México, ya desde el año 1901 en que Balbino Dávalos, en honor al Segundo Congreso Panamericano, tradujo al español aquel poema de Whitman ${ }^{45}$ que comienza:

Venid, yo haré que el Continente indisoluble sea 40

y el año 1902 en que José Juan Tablada, en un largo tributo a los poetas norteamericanos, insinuó en tin solo verso la misión libertadora de Whitman, describiéndole como

Un águila triunfando sobre una flor de lis, $\mathbf{4 7}$

habrá hasta nuestros días una procesión ininterrumpidà de poetas y pensadores, llámense José Vasconcelọs, Rafael Lozano, Mauricio Magdaleno, Rosendo Salazar, que aclaman a Whitman como creador y padre del arte contemporáneo, es decir, del arte posterior al modernismo. $\mathrm{Y}$ sería fácil nombrar a otros admiradores de Whitman que en Cuba, Colombia y Argentina, por ejemplo, se han identificado con la reacción contra el modernismo: José Manuel Poveda, de Cuba, que no logró escaparse por completo del movimiento que allí también se empezó a cultivar tardiamente por 1913; Rafael Maya, Jaramillo Medina, Rafael Vásquez, Antonio García, Jorge Padilla, de Colombia, que representan las nuevas tendencias poéticas de 1915 en adelante; y Luis L. Franco y Ricardo Güiraldes, de la Argentina, para citar sólo dos bien conocidos cultivadores de la poesía postmodernista en el Río de la Plata.

Si no hago mención especial de los ultraístas de las escuelas de vanguardismo de postguerra que acabaron definitivamente con el modernismo, es porque ellos al llevar a los últimos extremos al- 
gunas de las malas tendencias del movimiento, se apartaron aún más que los mismos modernistas del verdadero. Whitman - no importa que sus manifiestos todos anuncian al Gran Rebelde como uno de sus maestros predilectos, no importa que varias facetas de su llamada estética nueva parecen tener parentesco con la de Walt. $\mathrm{Si}$ a algunos de los poetas citados en este trabajo se les asocia a veces con el ultraismo, cabe advertir que aquella asociación fué una etapa relativamente corta $y$ de poco significado en el desarrollo de una obra total más sustancial y más en armonía con el espíritu de Whitman y de los postmodernistas en general.

Para terminar; cabe advertir que no hay que esperar encontrar en la obra de cada uno de los postmodernistas aquí mencionados, huellas tangibles y concretas de Whitman, ni mucho menos huellas que comparezcan iguales en la obra de todos. Cada uno descubre en Whitman lo que quiere descubrir, lo que más está en armonia con su modo peculiar de comprender lo que debe ser la nueva poesía: unos, como Sabat Ercasty, se sentirán más atraídos por su nota profética y sus impulsos cósmicos; otros, como Antonio Arráiz, por su conciencia americana y su espontaneidad natural y bárbara; y todavía otro, como el joven Neruda, por su exaltado egoísmo y su "jugosa animalidad sexual". Lo único que verdaderamente les une entre sí en su común reconocimiento de Whitman como el único pionero, o en las palabras de D. H. Lawrence, "the one man breaking a way ahead", queda bien expresado en esa frase de Walt que tanta verdad encierra tocante a su recepción e interpretación en cualquier parte del mundo:

The words of my book nothing, the drift of it everything. 48

Así lo comprendieron los postmodernistas que atendieron más a su inspiración que a sus palabras. Así para ellos como para los discipulos ingleses de Whitman de las últimas décadas del siglo pasado, ${ }^{40}$ Walt representaba ante todo el Gran Rebelde contra el statu quo, lo que ellos querían ser en mayor o menor grado con respecto al modernismo rezagado y desentonado de su época.

Es interesante notar otros paralelos entre estos postmodernistas y los ingleses a que me acabo de referir. En su gran mayoría los dos grupos estaban compuestos de jóvenes que ante el mismo umbral de su carrera literaria, respondieron más generosamente a 
los impulsos whitmanianos que los consagrados de la época, hecho que no le hubiera disgustado a Walt, que dijo una vez con respecto a su recepción en Inglaterra:

It's not the least flattering feature of my experience that $I$ have been most successful with young people, the justcomers, and least successful with the full done and over done masters of ceremony. ${ }^{50}$

$\mathrm{Y}$ finalmente, pocos de estos discípulos de Walt - séanlo ingleses, séanlo hispanoamericanos- han dejado una obra sólida y duradera. De los postmodernistas pocos son los conocidos, fuera de sus respectivos países; sólo unos cuàntos se salvarán para el futuro; en su gran mayoría quedarán - si ya no han quedado allá - atrás en la penumbra. Pero con toda justicia deberíamos recordar que a ellos les tocó nacer en una época de transición y de reacción, de poesía mediocre en comparación con la anterior. $\mathrm{Y}$ a ellos les tocó el papel gratuito de intentar forjar una nueva expresión estética; en Whitman encontraron la única voz capaz de conducirles hacia un arte más humano y más vital.

Estos versos que Punch escribió a la muerte de Whitman, ${ }^{51}$ que, según Blodgett, describen felizmente el caso del poeta en Inglaterra en la segunda mitad del siglo xIx, parécenme igualmente apropiados para el caso de Whitman en la América hispana de los años 1905-1920;

Whilst hearts are generous, and whilst woods are green, He shall find hearers, who in a slack time

Of puny bards and persimistic rhyme, Dared to bid men adventure and rejoice.

His "yawp barbaric" was a human voice;

The singer was a man.

JoHN E. ENGLEKIRK, Tulane University. 


\section{NOTA S}

1 Spanish III (Spanish-American) de la Modern Language Association of America, reunida en la ciudad de Washington, D. C., del 27 al 30 de diciembre de 1946.

2 "Notes on Whitman in Spanish America." Hispanic Reviev, v, 2, abril, 1938, pp. 133-138.

3 "Walt Whitman en Hispanoamérica", Revista Iberoamericana, vIII, 16, noviembre, 1944, pp. 343-356.

4 Walt Whitman. Buenos Aires, Americalee, 1945, 240.

5 Ibid.

6 Walt Whitman. Montevideo, Ceibo, 1944, $274 \mathrm{p}$.

7 "Walter Whitman, biblico, futurista poeta de América", Revista Iberoamericana, IV, 8, febrero; 1942, 305-314 pp.

8 Vida de Walt Whitman (The Magnificent Idler). La Plata, C. Calomino, 1942, 359 p. Trad. Marcos Fingerit.

9 Gigante americano (American Giant). Buenos Aires, Sudamericana, 1944. Trad. Luis Jiménez de Asúa. Pról. Dardo Cúneo.

10 Walt Whitman, Constructor para América (Walt Whitman, Builder for America). México, Séneca, 1942, 188 p. Trad. Rodolfo Usigli.

11 Ibid., 167-184 pp.

12 Canto a mi mismo. Buenos Aires, Losada, 1941, 199 p.

13 Véase mi "Bibliografía de obras norteamericanas en traducción española", Rezista Iberoamericana, vir, 16, noviembre, 1944, IX, 17, febrero, 1945 ; por otras traducciones de Whitman. Los números que se refieren a las antologías deben leerse: $3,4,14,15,16$.

14 Fué éste - "El nuevo mundo en busca de su expresión"- el tema del Tercer Congreso Internacional de Catedráticos de Literatura Iberoamericana, celebrado en Nueva Orleans del 21 al 24 de diciembre de 1942. Véase el trabajo leido ante el Congreso por Henry Seidel Canby, titulado "Who Speaks for New World Democracy", publicado en la Memoria del Tercer Congreso Internacional de Catedráticos de Literatura Iberoamericana. New Orleans, Tulane University Press, 1944, 195-203.

15 "La literatura hispanoamericana", Nosotros, LVIr, 219-220, agosto, 1927, 298 p. Cabe observar que Suárez Calimano sólo identifica a Whitman con la reacción que "iba contra la forma" modernista y con los "poetas civiles" de aquel entonces que llevaron "sti verso a las inquietudes sociales".

16 "De Verhaeren a Walt Whitman", La Pluma, I, 1, agosto, 1927, 4547 pp. 
17 Walt Whitman. Poemas. Valencia, Sempere, s. f., XIr.

18 Ibid.

19 Véase la nota anterior. Hay dos ediciones más recientes, la de Claudio García y Cía., Montevideo, publicada en 1939, y la de la Editorial Schapire, Buenos Aires, publicada en 1944. Esta salió con otro prólogo del traductor -además del prólogo original de la primera edición- titulado "Los discos poéticos de Walt Whitman", y con diez ilustraciones del pintor Carybé y dos fotografías fuera de texto.

20 Pereda Valdés, Ildefonso. Antologia de la moderna poesia uruguaya (1900-1927), Buenos Aires, El Ateneo, 1927, 101 p.

21 Pantheos. Montevideo, Bertani, 1917, 107 p.

22 Ibid., 103-104 pp.

23 Ibid., 128-129 pp.

24 Véase, por ejemplo, su poema "Canto al hombre esperado", Intemperie, Montevideo, Palacio del Libro, 1930.

25 Véase Vasseur, op cit.

26 Antonio S. Pedreira y Concha Meléndez. "Luis Lloréns Torres. El poeta de Puerto Rico", Revista Bimestre Cubana, xxxI, enero-junio, 1933, 330-352 pp.

27 Ibid.

28 Revista de las Antillas, junio de 1913, 93 p. Citado por Pedreira y Meléndez.

29 Del poema "Give me the splendid silent sun".

30 Pedro Juan Labarthe, en carta personal fechada el 29 de noviembre de 1946, que dice: "Lloréns declaró varias veces que Whitman le gustó nutucho, muchísimo, pero también declaró que no tuvo influencia suya. Yo creo que sí la tuvo, sin darse cuenta, el mismo don Luis."

31 Ibid. Dice Labarthe: "Si, sé que Whitman me encanta. Que lo comprendo hoy más que nunca, que vivo entre los suyos... Le leo a diario, como a Darío, como a Neruda y a Huidobro." Véase, además, su obra Cirios, San Júan, P. R., 1945, por su manifiesta admiración del "venerable Walt".

32 Confesión hecha a amigos mutuos y manifiesta en muchos versos suyos.

33 Dice Pedro Juan Labarthe en su Antología de poetas contemporáneos de 'Puerto Rico (México, Editorial Clásica, 1946, 87 p.), que "sus poetas preferidos son Rubén Darío, Jean Cocteau y Whitman".

34 "Prólogo", Aspero, 2a ed. Caracas, Edit. Elite, 1939, pp. 5-8. Reimpreso en Revista Nacional de Cultura, 1, 5, marzo, 1939, 31-32 pp.

35 Ibid.

36 Ibid. 
37 An Anthology of Contemporary Latin American Poetry, editada por Dudley Fitts, Norfolk, Conn., New Directions, 1942, 268-271 pp.

38 Cuba contemporánea, vIr, febrero, 1915, 198-208 pp.

39 Poetas norteamericanos. San José, C. R., García Monge, 129 p.

40 Véase su. "My debt to books", [Books Abroad 12: 2 (Spring, 1938), 167], donde confiesa: "The strongest literary influence in my later years has been Walt Whitman. Leaves of Grass revealed to me new horizons. AngloSaxon influence on Spanish poetry has been very slight and since our Spanish poetry has been quite conservative Whitman was a revelation to me. He has given the an example of freedom and virile art." $74 \mathrm{p}$.

41 Estudios de literatura chilena. 19 Serie. Santiago, Nascimento, 1938,

42 Fundada por Abraham Valdelomar acompañado de César Atahualpa Rodríguez, Percy Gibson, José Gálvez y otros.

43 Librería E. Rosay, s. f. (1916), 249 p. Contiene versos de Pablo Abril de Vivero, Hernán C. Bellido, Antonio Garland, Alfredo González-Prada, Federico Moore, Alberto Ulloa Sotomayor, Abraham Valdelomar, Félix del Valle.

44 En carta personal fechada el 13 de mayo de 1938.

45 "For you o Democracy."

46 Los grandes poetas norteamericanos. México, 1901, 15 p.

47 "Canto en honor de los poetas norteamericanos", El Sol (Buenos Aires), Iv, 155,15 de agosto de $1902,47 \mathrm{p}$.

48 "Shut not your Doors".

49. Véase Harold Blodgett. Walt Whitman in England. Ithaca, Cornell University Press, 1934, 215-218 pp.

50 Horace Traubel. With Walt Whitman in Camdem. New York, Mitchell Kennerley, 1915, vol. II, 181. Citado por Blodgett, 136.

519 de abril de 1892. 